\begin{tabular}{ccc}
\hline International Journal of Engineering \& Technology, 7 (2.7) (2018) $1017-1019$ \\
SPC & Website: www.sciencepubco.com/index.php/IJET \\
Research Paper & Technology \\
\hline
\end{tabular}

\title{
A Systematic Review on Integrity and Security in Adoption of Cloud Computing Technologies
}

\author{
Ramesh Kumar Mojjad $^{1 *}$, Dr. D.B.K.Kamesh ${ }^{2}$, K.B.K.S. Durga ${ }^{3}$, N Sainath ${ }^{4}$, A. Kavitha ${ }^{5}$ \\ ${ }^{1}$ Dept. of ECM, KL University, Vaddeswaram,Guntur(D), A.P. India \\ 2,3,4,5 Dept. of CSE, St. Martin's Engineering College, Hyderabad,Telangana \\ *Email: kameshdbk@gmail.com
}

\begin{abstract}
Distributed computing has waved the technological era in the year 2013 at a meeting in San Francisco, with sellers giving a lot of items and administrations that outfit IT which manages to convey request to cloud turmoil. Distributed computing pattern is expanding quickly for making the distributed computing well verse. The initial step for the association is to distinguish correct zone where the cloud issues exist. Surprisingly, the role of distributed computing has uplifted the era of business and government issues which lead to challenges towards security. The role of cloud technology improvements lead to display give business - supporting innovations in a proficient manner than any time in recent memory .the move from server to benefit based innovation got an intense change figuring innovation. However these improvements have made novel security challenges, including certain issues whose impact is a rising.[1] This paper displays a diagram and investigation of distributed computing, with a few security dangers, security issues, as of now utilized cloud innovations and security arrangements.
\end{abstract}

Keywords: Cloud services, Computing, cloud Models, Attacks, Technology, Security services, Models.

\section{Introduction}

Distributed computing is a group of services that are dispensed as per demand. Distributed computing provides efficient approaches to support administrations [1] which are new, imaginative, effective and to change the manner of business style. Distributed computing is well known supreme figuring innovation. With a bundle of assets and overhauled gave.

The cloud specialists with the help of web performed the cloud administrations sited throughout the world. Distributed computing makes it easy for the clients to utilize the virtual assets by means of web tools. Distributed computing has got in to the spotlight in the couple of years by the various Cloud services. Various providers of cloud service are Amazon, Google Engine, IBM, Oracle Cloud, Office 365. The Era of

distributed computing is upgrading fast, a wide variety of security issue [2] arise and are creating challenges before the clients and suppliers.

\section{Cloud Service Models}

Cloud provides services such as Platform as a Service - PAAS which is used to acquire the product and its capacity with internet. programming presents institutions to get into enterprise usefulness an ease ordinarily no longer as much as paying for legal programs in perception of the way to expenses month to month fee. the product is facilitated remotely customers need not extra equipment. Software - as-a-service dispenses with the all opportunities of business enterprise particles to deal with the establishment \& set - up, every day safeguarding, guide. PAAS provides the ability to the customers to send to the cloud framework. [3] PAAS display, cloud companies procures a figuring stage, usually comprising running gadget, programming language execution surroundings, database and many servers.

\section{Cloud Deployment Models}

Open Cloud- It is defined as open cloud that gives a public access system which can be used by everyone. The advantages of Open cloud are taken as a compensation for effective utilization of the services. A private cloud is a foundation that provides the services, a unique association, with as insider or an outsider and the cloud which is placed remotely is regarded as "remotely facilitated" private cloud and the third facilitated to get- together which are named as "on introduce" private service cloud. [4]

\section{Cloud Computing Technologies}

\subsection{Microsoft Cloud Technologies}

Microsoft is the most advantageous supplier of cloud advancements and applications with consequences and suits varieties of commercial enterprise needs. It supplies all sorts of administrations whether it is a paas, iaas or saas. Microsoft gives the windows server and framework jog and within the occasion of platform-as-a-service, it give windows azure, which can support manufacture, host and scale packages in Microsoft center without earlier cost and recompense only that you use .[5] Paas administrations are sqlserver and visual studio where as the office 365 , proportion-factor servers, dynamic crm and trade server are the software - as-a-services gave the aid of Microsoft and could confirm that it is providing the complete package on your business.

\subsection{Oracle Cloud Technologies}

Prophet offers general undertaking study open cloud association such as iaas, paas and saas. From this you absolutely possess 
awareness in your business enterprise without agonizing the management. prophet provides the following utilites [6] database, it's far procured database-as-a-transporter along entering in to the database inside the cloud straightforwardly with big tool establishments, or as a platform as a company, with whole development and forwarding surroundings. we can generate income through its services as a unique pattern based totally administration, or a digital device with an organized, strolling oracle database existence for utilizing the prophet cloud database you virtually want to generate a document with significant e mail identification and login with the given credentials that may appreciate the management for offering a 30 days trial as per your need.

prophet java cloud benefit as this administration it offers you the software development and basis and management gadgets, you may create j2ee requirements jsp, jsf, serve allow, ejb, jpa, jax rs and jax-ws applicator particles .you can run outstanding systems like spring, hibernate and create for your decision of cloud enabled ide, as an example, oracle $\mathrm{j}$ developer, eclipse and net beans. and ultimate but no longer the minimal internet good judgment server as an utility server.prophet transportable cloud -it is a simple assignment ordeal availability, it gives you resultseasily named interfaces, crowd apis and gather versatile packages to your undertaking frameworks. versatile cloud gives you can more workplaces, for instance, transportable apps, notifications (email, sms, voice) and data regulate

prophet cloud archive and prophet allotted storage -it offers you a simple and managed cloud based file sharing and coordinated attempt association stable safety. and prophet dispensed garage workplace offer you a strong and at ease statistics stockpiling level for putting away and getting to statistics from wherever associated with net. provide the factors like reinforcement, sharing, sparing and appropriating records among application and clients without a hassle.

prophet cloud informing oracle cloud informing management empowers framework that make a speak particle hyperlink among programming segments with the office of sending and getting messages via single informing api and make a dynamic motorize business workflow ecosystem[8].

\subsection{Cloud Technologies provided by Google}

Google cloud additionally offers the administrations, for instance, software - as-a- carrier, platform-as-a-providerand infrastructureas-a-provider. google cloud empowers designers to gather, check and send applications on google's quite adaptable and comfortable framework. as we recognize that google has as of now gave framework that allows google to go back billions of question items in milliseconds, provide stockpiling to around 425 million gmail clients and serve 6 billion hours of youtube video every month. google can fabricate, compose and paintings a fantastic system of servers and fiber-optic hyperlinks .all this in total makes google the king of all cloud.

google apps engine-with apps engine you could run your programs on a completely oversaw platform-as-a-service utilizing worked in administrations. here you can compose packages in likely the most widely recognized programming dialects, for instance, java, php and python.

\subsection{Cloud Computing Security Threats}

Cloud computing faces as much security threats as that are existing in the networks, intranets. These threats come in various forms. Cloud computing alliance did research in 2013 on cloud computing security threats and identified these threats like Traffic Hijacking, Insecure Interface and APIs, Denial of Service, Malicious Insiders, Abuse of Cloud Services, Shared Technology Vulnerabilities and Data Breaches etc.

\subsection{Cloud Security Issues}

While cost and usability are the two primary solid advantages of the distributed computing, there are some major disturbing issues that should be referenced while permitting moving basic application and touchy information to open and shared cloud environment. The principle viewpoint depicting the accomplishment of any new registering innovation is the tallness of security it gives whether the information situated in the cloud is ensured at that level that it can maintain a strategic distance from any kind of security issue. So we should state that Security and protection are the key difficulties in the distributed computing. Here are some security issues, we have introduced in this paper.

Data classification issue: Confidentiality is an arrangement of guidelines or an assertion that limits get to or area confinement on specific sorts of data so in cloud information live publically so Confidentiality alludes to, customer's information and calculation assignment are to be kept secret from both cloud supplier and different clients who is utilizing the administration. We should ensure that user's private or secret information particle ought not to be gotten to by anybody in the distributed computing framework, including application, platform, CPU and physical memory. Unmistakably user's private information is revealed to specialist organization on the following circumstance as it were.

Situation 1. The first circumstance where user's information particle might be unveiled when specialist organization knows where the user's private data lives in the cloud frameworks.

Situation 2. The second situation where user's information particle might be uncovered when specialist co-op has the expert to get to and accumulate user's private data in the cloud frameworks

Situation 3. The third situation where user's information particle might be unveiled when expert organization could be identifying significance of user data particle within the cloud outlines.

These are providing the situation as professional business enterprise can group or acquire consumer's information or facts and if the administrator provider should recognize region with records within the allotted computing and feature the professional to load on to the customer's facts that we recognize that the existing allotted computing incorporates of different layers with a program and they are software layer, platform layer and infrastructure layer. Product layer provides the uses for the purchaser by running on the cloud framework. the stage layer provides the level. The muse layer offers the gadget belongings to registering, stockpiling and machine. in spite of the truth that as the each expert organization has its very own unique programming, stage and basis layer with this when purchaser makes use of the cloud software gave with the aid of expert business enterprise, it is obligatory for the client to make use of the stage and moreover framework gave by the expert organization and on this way specialist organization is aware of about, where the person's records is put and the overall openness to the statistics

\subsubsection{Data accessibility issue}

Storing the data at remote area that is claimed by others. If the cloud stops working then the information cannot be accessible as the information relies on upon single specialist services. Vulnerabilities to the data accessibility are flooding attacks that causes preventing from securing supervision and Direct/Indirect (DOS) service. Distributed computing is to give on - demand supervision of various ways. If a specific service is no longer accessible for the customer then it can't hold the Service Level Agreement (SLA) and the clients decline the confidence in the 
framework of cloud.

\subsubsection{Data respectability issue}

It defines the "fulfillment" and "wholeness" of the data which is the primary and essential need of the data innovation, Trustworthiness of data is vital in the database and also information stockpiling is vital and essential component in the cloud and it is the primary component that can alter cloud execution. The data trustworthiness provides a proof for the consistency and legitimacy with normality of data. It is a best method to secure the information and information stockpiling which can be recovered easily in a specified format Consequently, the distributed storage is acting as the main component for maintaining the data regularly.

\subsubsection{Information security issue}

when we explain the data stockpiling among the distributed computing or on preface applicator and data organization to demonstrate, the regular data of each venture keeps on dwelling inside the undertaking limit and is center to its physical, consistent and staff security and get to control rules. Although in Softwareas- a-Service model or open cloud the undertaking information is put away outside the venture limit, by the CSP. So therefore, the CSP must consent to execute extra security checks to guarantee information security and need to avoid breaks because of security vulnerabilities in the application or through malignant employees. These all above concern issues require to utilize a solid encryption systems for the assurance of the information on the grounds that the some conventional encryption which have been utilized since, are not as intense as we need. The information assurance should be devil alimented keeping in mind the end goal to secure information from the accompanying instabilities.

\subsubsection{Trust issue}

Trust is a worthy issue in distributed computing. Trusting the spin around "assurance" and conviction of the entities, their data \& objects, information particles that performs in an anticipated way. Trust has to generated in between human to human and machine to machine and also in between human to machine or machine to human. Consequently in distributed computing whenever a client wants to store information on distributed storage, they need to trust the cloud supplier so that they should not hesitate store their information on cloud as similarly we utilize Gmail and other types of servers.

\section{Solutions To Incorporate Security Vulnerabilities In Cloud Computing}

\subsection{Configure Support}

When clients store th information in the given cloud they do not have the data where the information is put away. Along these lines cloud specialist co-op must give review instruments to the clients to look at manage how there is put away, ensured, utilized and confirm approach demon alimentation.

\subsection{Recovery office}

Cloud supplier must give sheltered and supportive recuperation office, so in any circumstance if information is lost as a result beacause of any cause, information could be recouped such that coherence information could be overseen.

\subsection{Back up office}

Common debacle may mischief or harms physical gadgets that might be the reason of information misfortune. Thusly to maintain a strategic distance from this issue merchant must give the reinforcement of information particle, this office gives a key affirmation of administration gave by specialist co- ops.

\subsection{Encryption calculation}

We that cloud specialist co-op scramble users information utilizing a solid encryption system however at some scenario the encryption mis chances can generate information totally futile and at the opposite side encryption additionally confuses the accessibility of information. To take care of this testing issue cloud supplier must give verification that encryption system was outline and appropriately tried by proficient and experience expert.

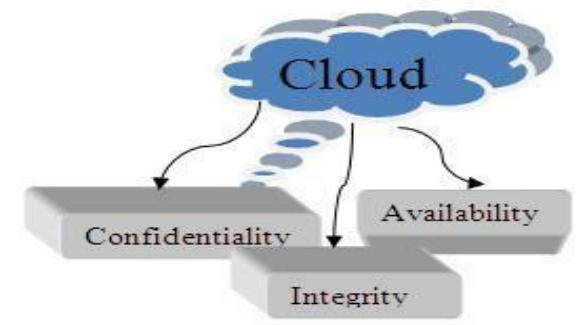

\section{Conclusion}

Distributed computing is the powerful innovation which is cost effective, time and execution. Ultimately the use of distributed computing increases a lot in the coming years. In this paper, the need and necessity of distributed computing and its security issues were discussed where some of the issues are the key worry in the distributed computing along with protection and honesty of information.

\section{References}

[1] Juneja, Gurpreet K. "Use of Modeling Language to deploy applications in clouds." Dhiwar, Kamlesh Kumar. "Aspect of Cloud computing."

[2] Akintomide, O. A. "Cloud computing: The third revolution in IT." Library Progress (International) 33.1 (2013): 77- 94

[3] Mell, Peter, and Timothy Grance. "The NIST definition of cloud computing (draft)." NIST special publication 800.145 (2011): 7.

[4] Pearson, Siani, Yun Shen, and Miranda Mowbray. "A privacy manager for cloud computing." Cloud Computing. Springer Berlin Heidelberg, 2009. 90-106.

[5] T OGRAPH, B., and Y. RICHARD MORGENS."Cloud computing."Communications of the ACM 51.7 (2008).

[6] Velte, Toby, Anthony Volte, and Robert Elsenpeter. Cloud computing, a practical approach. McGraw-Hill, Inc., 2009. 